\title{
The Clinicopathological Characteristics of Henoch-Schönlein Purpura Nephritis with Presentation of Nephrotic Syndrome
}

\author{
Jiaxing Tan ${ }^{\mathrm{a}, \mathrm{b}} \quad$ Yi Tang $^{\mathrm{a}} \quad$ Yicong $\mathrm{Xu}^{\mathrm{b}}$ Siyu Yan ${ }^{\mathrm{b}}$ Yuanyuan $\mathrm{Xu} \mathrm{u}^{\mathrm{b}}$ \\ Li Tan ${ }^{a}$ b Zhengxia Zhong ${ }^{a, b}$ Padamata Tarun ${ }^{b} \quad$ Wei Qin ${ }^{a}$ \\ aDivision of Nephrology, Department of Medicine, West China Hospital, Sichuan University, \\ Chengdu, China; ${ }^{b}$ West China School of Medicine, Sichuan University, Chengdu, China
}

\section{Keywords}

Henoch-Schönlein purpura nephritis · Adult onset · Nephrotic syndrome ·

Clinicopathological manifestations $\cdot$ Renal outcome

\begin{abstract}
Background: Henoch-Schönlein purpura nephritis (HSPN) is a common vasculitis involving the kidneys, with a lower incidence in adults. Meanwhile, nephrotic syndrome (NS) can appear in HSPN. However, the clinicopathological features and renal outcome of adult-onset HSPN presenting with NS (NS-HSPN) have not been well clarified. Methods: A total of 191 HSPN patients were prospectively analyzed and comparisons were made between NS-HSPN and non-NSHSPN. Multivariate Cox regression analysis was carried out to find the unfavorable factors of renal outcome of NS-HSPN. Results: Among the 191 patients, 44 (23.0\%) had NS-HSPN. Apart from edema and abdominal pain, patients with NS-HSPN tended to have lower levels of erythrocytes and hemoglobulin in blood as well as a greater number of erythrocytes in urine $(p<$ 0.05). Mesangial proliferation was the most common pathological lesion in HSPN and the rates of crescent formation were significantly different, with $54.5 \%$ in NS-HSPN and $33.3 \%$ in non-NSHSPN $(p<0.05)$. Notably, 18.2 and $4.8 \%$ of patients reached the composite endpoints in the NSHSPN and non-NS-HSPN groups, respectively $(p<0.05)$, demonstrating that NS-HSPN patients were more likely to progress to end-stage renal disease and had a worse outcome. We also found that hypertension, estimated glomerular filtration rate (eGFR), cystatin, and tubular atrophy/interstitial fibrosis (HR $>1, p<0.05)$ at onset were correlated with adverse outcome in NSHSPN. Conclusion: NS-HSPN had more severe clinicopathological manifestations and poorer prognosis. The adverse predictors of NS-HSPN principally depend on clinicopathological presentation rather than on different therapies, and hypertension, eGFR, cystatin, and tubular atrophy/interstitial fibrosis can serve as independent risk factors in NS-HSPN.
\end{abstract}




\section{Kidney \\ Blood Pressure \\ Research}

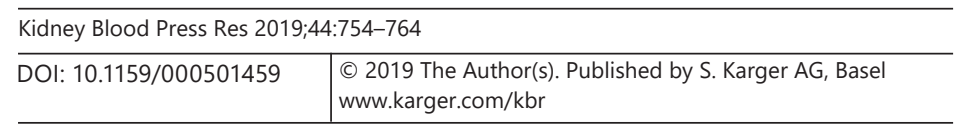

Tan et al.: The Nephrotic Syndrome of Henoch-Schönlein Purpura Nephritis

\section{Introduction}

Henoch-Schönlein purpura (HSP) is a common vasculitis predominantly affecting arterioles, venules, and capillaries [1]. The clinical manifestations of HSP include palpable purpuric rashes, diffuse abdominal pain with or without gastrointestinal bleeding, arthralgia, gross hematuria, and proteinuria [2]. It is generally acknowledged that HSP is a self-limiting disease while the long-term prognosis is principally based on renal involvement, known as HSP nephritis (HSPN). Owing to lower incidence in adults, there is little high-quality research on adult-onset HSPN.

Nephrotic syndrome (NS) is a collection of manifestations including massive proteinuria and hypoalbuminemia, commonly with swelling and/or hyperlipidemia [3]. In clinical practice, we find that NS is relatively less frequent in HSPN; its effect on prognosis has been poorly studied, though it has been proved that NS is associated with unfavorable outcomes in many kidney diseases, such as immunoglobulin A nephropathy, and can indicate severe renal histopathological changes [4]. Consequently, this study was carried out to get more acquainted with the clinicopathological characteristics and prognosis of adult patients suffering from HSPN presenting with NS (NS-HSPN).

\section{Materials and Methods}

\section{Inclusion and Exclusion Criteria}

In this prospective study, 209 patients with HSPN who had undergone a renal biopsy were recruited from the West China Hospital of Sichuan University in China between October 2010 and June 2017. The diagnosis of HSPN was made when HSP patients had microscopic or gross hematuria and/or proteinuria, further confirmed by renal biopsy [5]. The proposals based on the American College of Rheumatology database and methodology were used as diagnostic criteria of HSP [6]. All patients enrolled in our study were over 14 years old. Nevertheless, patients with other systemic diseases such as liver cirrhosis, malignant neoplasm, systemic lupus erythematosus, etc. were not considered. Additionally, patients whose demographic and laboratory data were not sufficient for our analysis were also excluded. Accordingly, 191 patients with HSPN were analyzed in our study.

\section{Data Collection}

General and clinical characteristics were reviewed including age, sex, follow-up duration, time to endpoint, clinical symptoms, systolic blood pressure, diastolic blood pressure, and treatment strategies. Laboratory data comprised excretion levels of 24-h proteinuria, number of erythrocytes in urine (u-RBCs) at high magnification, serum albumin, serum creatinine, uric acid, triglycerides, cholesterol, low-density lipoprotein cholesterol (LDL-C), alanine aminotransferase, aspartate aminotransferase, hemoglobin, platelets, erythrocytes, and leukocytes in blood. Renal pathology data included mesangial proliferation, endocapillary proliferation, segmental glomerulosclerosis, tubular atrophy, interstitial fibrosis, and crescents.

\section{Treatment Regimens}

Four pharmacotherapeutic schemes were widely used clinically, including angiotensinconverting enzyme inhibitor (ACEI)/angiotensin receptor blocker (ARB) (scheme A), glucocorticoids (scheme B), glucocorticoids combined with immunosuppressants (scheme C), and pulse therapy with methylprednisolone and cyclophosphamide (scheme D). The choice of scheme depended on the severity of disease and the doctor's experience. Patients in scheme A received full-dose ACEI or ARB alone. Patients in scheme B received oral glucocorticoids 


\section{Kidney \\ Blood Pressure \\ Research}

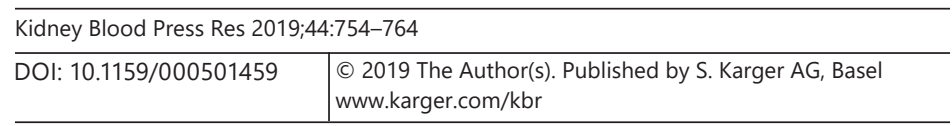

Tan et al.: The Nephrotic Syndrome of Henoch-Schönlein Purpura Nephritis

daily (0.5-1 mg/kg prednisone) and tapered down gradually. Glucocorticoids combined with immunosuppressive agents, including cyclophosphamide, mycophenolate mofetil, azathioprine, leflunomide, tacrolimus, and cyclosporin, were used in scheme C $[7,8]$. Patients in scheme D were treated with intravenous pulse methylprednisolone followed by oral glucocorticoids and sequentially treated with intravenous cyclophosphamide. Optimal renal protective treatments were used in all schemes.

\section{Definitions and Outcome Measurements}

The definition of NS was hypoalbuminemia $<30 \mathrm{~g} / \mathrm{L}$ and proteinuria $>3.5 \mathrm{~g} / \mathrm{day}$. The diagnosis of hypertension was based on standards of the World Health Organization Expert Committee $(>140 / 90 \mathrm{~mm} \mathrm{Hg})$. Hematuria was defined as u-RBCs $>5$ at high power. The Chronic Kidney Disease Epidemiology Collaboration formula was used to calculate the estimated glomerular filtration rate (eGFR).

It has been well accepted that immunoglobulin A nephropathy and HSPN share similar pathogenesis and pathology findings. Owing to no standards in pathological classification of adult-onset HSPN, the Oxford classification might be effective to predict the long-term outcomes of HSPN [9]. The definitions of mesangial proliferation, endocapillary proliferation, segmental glomerulosclerosis, tubular atrophy, and interstitial fibrosis are largely referred to the Oxford classification [10-12]. Moreover, crescents were a feature in HSPN which could not be ignored. Therefore, we also collected the pathological data of crescents [13].

The primary endpoint was the composite of a 50\% decline in eGFR, end-stage renal disease (ESRD) and/or death. The definition of ESRD was eGFR $<15 \mathrm{~mL} / \mathrm{min} / 1.73 \mathrm{~m}^{2}$ or maintenance renal replacement treatment. Secondary renal outcome was classified as complete remission, partial remission, active renal disease, and renal insufficiency, based largely upon Meadow's criteria [14]. Complete remission was defined as 24-h proteinuria $\leq 0.3 \mathrm{~g}$, with no hematuria and normal renal function. Partial remission was defined as 24-h proteinuria $\leq 1 \mathrm{~g}$, with or without recurrent hematuria. Active renal disease was defined as 24-h proteinuria $>1 \mathrm{~g}$ or a decreased eGFR level $>10 \%$. The definition of renal insufficiency was eGFR $<60 \mathrm{~mL} / \mathrm{min} / 1.73 \mathrm{~m}^{2}$.

\section{Statistical Analyses}

Data were analyzed using the IBM SPSS Statistics 22.0 software. Continuous variables were expressed as mean \pm standard deviation or median with interquartile range, which were compared by Student $t$ test or Wilcoxon test. Categorical variables were summarized as numbers and percentages, which were compared by $\chi^{2}$ test or Fisher exact test. Kaplan-Meier estimation was performed to calculate the survival outcome and rate of endpoint. The crude effects of clinical manifestations and pathological features on renal outcomes were evaluated by multivariate Cox regression analysis. Additionally, all $p$ values were two-tailed and statistical significance was reached when $p$ was $<0.05$.

\section{Results}

\section{Demographic Data}

A total of 209 patients with HSPN were registered in our medical center, whereas only 191 patients were recruited in our study because of the exclusion criteria. Forty-four patients (23 male and 21 female) were categorized into the NS-HSPN group, while 147 patients (64 male and 83 female) were assigned to non-NS-HSPN group (Fig. 1). Table 1 reveals the demographic data. The age of patients at onset ranged from 14 to 76 years, with a median of 24 years, and the mean follow-up of our cohort was 27 months. 


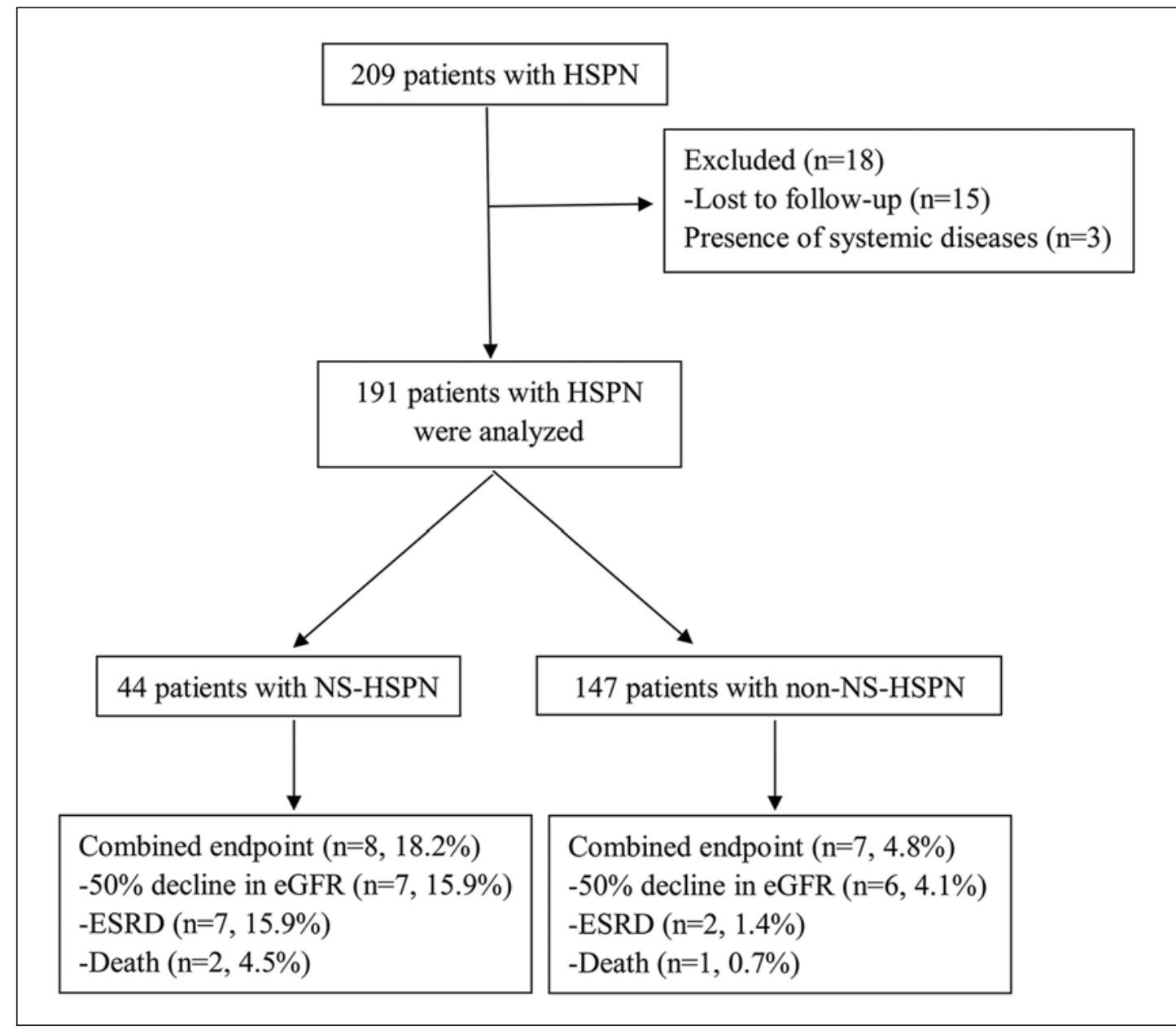

Fig. 1. Flow diagram of patient progress and outcomes. eGFR, estimated glomerular filtration rate; ESRD, end-stage renal disease; HSPN, Henoch-Schönlein purpura nephritis; NS-HSPN, Henoch-Schönlein purpura nephritis presenting with nephrotic syndrome.

\section{Clinicopathological Findings}

As presented in Table 1, edema $(65.9 \%, p<0.01)$ and abdominal pain $(40.9 \%, p<0.05)$ were significantly more common in the NS-HSPN group $(p<0.05)$, and patients with NS seemed to have higher levels of systolic blood pressure (127.84 $\pm 20.39 \mathrm{~mm} \mathrm{Hg}, p=0.078)$ and rates of bloody stools $(20.5 \%, p=0.099)$. However, no significances were found in age at onset, sex, purpura, gross hematuria, arthralgia, diastolic blood pressure, mean arterial pressure, and hypertension $(p>0.1)$. There were obviously different laboratory findings between those two groups at baseline. Apart from massive proteinuria and hypoalbuminemia, patients with NS-HSPN tended to have lower levels of erythrocytes $\left(4.52,3.92-4.75 \times 10^{12} / \mathrm{L}\right.$, $p<0.01)$ and hemoglobulin $(123.14 \pm 19.69 \mathrm{~g} / \mathrm{L}, p<0.01)$ in blood as well as a greater number of u-RBCs $(103,13-320 /$ high-power field, $p<0.05)$ and platelets $\left(260.30 \pm 106.14 \times 10^{9} / \mathrm{L}\right.$, $p<0.05)$. In addition, median cholesterol was $6.53 \mathrm{mmol} / \mathrm{L}(4.53-7.48 \mathrm{mmol} / \mathrm{L})$ in the NS-HSPN group and $4.80 \mathrm{mmol} / \mathrm{L}(3.94-5.78 \mathrm{mmol} / \mathrm{L})$ in the non-NS-HSPN $(p<0.05)$. LDL-C was $3.47 \mathrm{mmol} / \mathrm{L}(2.59-5.16 \mathrm{mmol} / \mathrm{L})$ versus $2.62 \mathrm{mmol} / \mathrm{L}(2.11-3.41 \mathrm{mmol} / \mathrm{L}, p<0.05)$, respectively. However, we did not find any differences in creatinine, eGFR, uric acid, triglyceride, alanine aminotransferase, aspartate aminotransferase, cystatin, and leukocytes. 
Table 1. Clinical manifestations and laboratory data of HSPN patients at baseline

\begin{tabular}{|c|c|c|c|}
\hline & NS-HSPN $(n=44)$ & Non-NS-HSPN $(n=147)$ & $p$ values \\
\hline Age at diagnosis, years & $21(16-43)$ & $27(18-44)$ & 0.657 \\
\hline Male/female (\%) & $23 / 21(52.3 / 47.7)$ & $64 / 83(43.5 / 56.5)$ & 0.307 \\
\hline Skin purpura (\%) & $41(93.2)$ & $135(91.8)$ & 1.000 \\
\hline Edema (\%) & $29(65.9)$ & 44 (29.9) & $0^{* *}$ \\
\hline Gross hematuria (\%) & $3(6.8)$ & $19(12.9)$ & 0.419 \\
\hline Arthralgia (\%) & $11(25.0)$ & $29(19.7)$ & 0.451 \\
\hline Abdominal pain (\%) & $18(40.9)$ & $36(24.5)$ & $0.034^{*}$ \\
\hline Bloody stools (\%) & $9(20.5)$ & $16(10.9)$ & 0.099 \\
\hline Follow-up, months & $18(12.0-50.0)$ & $19.5(12.0-34.5)$ & 0.423 \\
\hline $\mathrm{SBP}, \mathrm{mm} \mathrm{Hg}$ & $127.84 \pm 20.39$ & $122.20 \pm 17.93$ & 0.078 \\
\hline $\mathrm{DBP}, \mathrm{mm} \mathrm{Hg}$ & $80.7 \pm 13.60$ & $78.76 \pm 11.34$ & 0.343 \\
\hline MAP, mm Hg & 95.67 (84.33-106.33) & 93.33 (86.59-102.67) & 0.205 \\
\hline Hypertension (\%) & $14(31.8)$ & $32(21.8)$ & 0.171 \\
\hline Proteinuria, g/24 h & $5.40(3.84-8.25)$ & $1.65(0.80-2.76)$ & $0^{* *}$ \\
\hline $\mathrm{u}-\mathrm{RBCs} / \mathrm{HPF}$ & $103(13-320)$ & 25 (8-109) & $0.021^{*}$ \\
\hline Albumin, g/L & $24.24 \pm 4.79$ & $38.54 \pm 5.61$ & $0^{* *}$ \\
\hline $\mathrm{SCr}, \mu \mathrm{mol} / \mathrm{L}$ & $72.30(56.90-111.10)$ & $70.25(57.95-86.70)$ & 0.112 \\
\hline Uric acid, $\mu \mathrm{mol} / \mathrm{L}$ & $356.92 \pm 113.49$ & $337.09 \pm 99.89$ & 0.265 \\
\hline $\mathrm{eGFR}, \mathrm{mL} / \mathrm{min} / 1.73 \mathrm{~m}^{2}$ & $112.80(71.50-131.15)$ & $110.55(87.13-129.65)$ & 0.525 \\
\hline Triglycerides, mmol/L & $1.93 \pm 0.92$ & $1.77 \pm 1.01$ & 0.341 \\
\hline Cholesterol, mmol/L & $6.53(4.53-7.48)$ & $4.80(3.94-5.78)$ & $0^{* *}$ \\
\hline LDL-C, mmol/L & $3.47(2.59-5.16)$ & $2.62(2.11-3.41)$ & $0 * *$ \\
\hline ALT, IU/L & $26.59 \pm 18.25$ & $28.46 \pm 23.28$ & 0.627 \\
\hline AST, IU/L & $23.59 \pm 10.13$ & $22.59 \pm 8.30$ & 0.405 \\
\hline Cystatin C, mL/L & $1.38 \pm 0.72$ & $2.90 \pm 15.47$ & 0.517 \\
\hline RBCs, $\times 10^{12} / \mathrm{L}$ & $4.52(3.92-4.75)$ & $4.69(4.31-5.14)$ & $0.002^{* *}$ \\
\hline Hemoglobin, g/L & $123.14 \pm 19.69$ & $138.05 \pm 18.91$ & $0^{* *}$ \\
\hline Platelets, $\times 10^{9} / \mathrm{L}$ & $260.30 \pm 106.14$ & $221.54 \pm 90.20$ & $0.018^{*}$ \\
\hline WBCs, $\times 10^{9} / \mathrm{L}$ & $9.62(6.78-13.74)$ & $8.88(6.15-11.80)$ & 0.151 \\
\hline
\end{tabular}

ALT, alanine aminotransferase; AST, aspartate aminotransferase; DBP, diastolic blood pressure; eGFR, estimated glomerular filtration rate; HPF, high-power field; HSPN, Henoch-Schönlein purpura nephritis; LDL-C, low-density lipoprotein cholesterol; MAP, mean arterial pressure; NS-HSPN, Henoch-Schönlein purpura nephritis presenting with nephrotic syndrome; RBCs, erythrocytes; SBP, systolic blood pressure; $\mathrm{SCr}$, serum creatinine; u-RBCs, erythrocytes in urine; WBCs, leukocytes. ${ }^{*} p<0.05$, ${ }^{* *} p \leq 0.01$.

As presented in Table 2, the most frequent renal pathological lesions in the NS-HSPN group were mesangial proliferation $(39 / 44,88.6 \%)$ and crescents $(24 / 44,54.5 \%)$, while in the non-HSPN group, mesangial proliferation $(122 / 147,83.0 \%)$ and tubular atrophy/interstitial fibrosis $(62 / 147,42.2 \%)$ were the most common lesions. There were remarkable differences in crescent formation, with $24 / 44$ (54.5\%) and 49/147 (33.3\%) in the NS-HSPN and non-NS-HSPN group, respectively. There were no other significant histopathological characteristics between the two groups.

\section{Treatments}

Table 3 shows the principal treatment regimens for the 191 patients enrolled in our study. No patients with NS-HSPN and only $11.6 \%$ of non-NS-HSPN patients were treated with ACEI or ARB only. The proportion of glucocorticoids alone, glucocorticoids combined with immunosuppressants, and pulse therapy with methylprednisolone and cyclophosphamide was $25.0,47.7$, and $27.3 \%$ in the NS-HSPN group and $44.2,33.3$, and $10.9 \%$ in non-NS-HSPN 
Table 2. Renal pathological findings of HSPN patients

\begin{tabular}{llcl}
\hline & NS-HSPN $(n=44)$ & Non-NS-HSPN $(n=147)$ & $p$ values \\
\hline M1/M0 (\%) & $39 / 5(88.6 / 11.4)$ & $122 / 25(83.0 / 17.0)$ & 0.481 \\
E1/E0 (\%) & $11 / 33(25.0 / 75.0)$ & $21 / 126(14.3 / 85.7)$ & 0.095 \\
S1/S0 (\%) & $15 / 29(34.1 / 65.9)$ & $54 / 93(36.7 / 63.3)$ & 0.749 \\
C1/C0 (\%) & $24 / 20(54.5 / 45.5)$ & $49 / 98(33.3 / 66.7)$ & $0.011^{*}$ \\
C0/0-25/>25 (\%) & $20 / 10 / 14(45.5 / 22.7 / 31.8)$ & $97 / 39 / 11(66.0 / 26.5 / 7.5)$ & $0^{* *}$ \\
T1/T0 (\%) & $15 / 29(34.1 / 65.9)$ & $62 / 85(42.2 / 57.8)$ & 0.337 \\
\hline
\end{tabular}

"1" stands for presence, "0" stands for absence. C, crescents; E, endocapillary proliferation; HSPN, HenochSchönlein purpura nephritis; M, mesangial proliferation; NS-HSPN, Henoch-Schönlein purpura nephritis presenting with nephrotic syndrome; S, segmental glomerulosclerosis; T, tubular atrophy/interstitial fibrosis. ${ }^{*} p<0.05,{ }^{* *} p \leq 0.01$.

Table 3. Principal treatments of patients with HSPN

\begin{tabular}{llll}
\hline & $\begin{array}{l}\text { NS-HSPN } \\
(n=44)\end{array}$ & $\begin{array}{l}\text { Non-NS-HSPN } \\
(n=147)\end{array}$ & $p$ values \\
\hline ACEI/ARB (\%) & $0(0)$ & $17(11.6)$ & $0.014^{*}$ \\
Glucocorticoids (\%) & $11(25.0)$ & $65(44.2)$ & $0.022^{*}$ \\
Glucocorticoids + immunosuppressants (\%) & $21(47.7)$ & $49(33.3)$ & 0.082 \\
Pulse therapy with MP and CTX (\%) & $12(27.3)$ & $16(10.9)$ & $0.007^{* *}$ \\
\hline
\end{tabular}

ACEI, angiotensin-converting enzyme inhibitor; ARB, angiotensin receptor blocker; CTX, cyclophosphamide; HSPN, Henoch-Schönlein purpura nephritis; MP, methylprednisolone; NS-HSPN, Henoch-Schönlein purpura nephritis presenting with nephrotic syndrome. * $p<0.05$, ** $p \leq 0.01$.

group, respectively. On the whole, NS patients were treated with more aggressively immunosuppressive treatments.

\section{Outcomes}

In our cohort, $7.9 \%$ of patients with HSPN progressed to ESRD. Kaplan-Meier survival analysis, shown in Figure 2, indicated that 18.2\% (8/44) and 4.8\% (7/147) patients reached the combined endpoints in the NS-HSPN and non-NS-HSPN group, respectively $(p<0.05)$. Further information on laboratory data from the last follow-up is provided in Table 4. Comparison between NS-HSPN and non-NS-HSPN patients demonstrated significant differences in creatinine and eGFR $(p<0.05)$. However, statistical significances were not found in proteinuria, u-RBCs, albumin, uric acid, triglyceride, cholesterol, or LDL-C. Notably, patients with NS-HSPN tended to have a higher proportion of renal insufficiency $(22.7$ vs. $10.9 \%, p<0.05)$ and a lower rate of partial remission (20.5 vs. $37.4 \%, p<0.05$ ), whereas other renal outcomes including rates of complete remission ( 27.3 vs. $25.2 \%, p=0.779$ ) and active renal disease (29.5 vs. $26.5 \%, p=0.693$ ) showed no differences.

\section{Factors of Renal Outcome}

Multivariate Cox regression analysis was applied to explore the risk factors in NS-HSPN patients, for the reason that more patients with NS-HSPN would progress to the combined 
Table 4. Laboratory data at the last follow-up

\begin{tabular}{lccl}
\hline & NS-HSPN $(n=44)$ & Non-NS-HSPN $(n=147)$ & $p$ values \\
\hline Proteinuria, g/24 h & $0.31(0.15-0.81)$ & $0.30(0.19-0.70)$ & 0.129 \\
u-RBCs/HPF & $41.89 \pm 146.13$ & $25.16 \pm 84.23$ & 0.340 \\
Albumin, g/L & $42.81 \pm 5.74$ & $44.20 \pm 4.67$ & 0.102 \\
SCr, $\mu$ mol/L & $75.40(62.70-116.10)$ & $69.05(59.15-92.17)$ & $0.014^{*}$ \\
Uric acid, $\mu$ mol/L & $371.80 \pm 113.30$ & $357.17 \pm 92.02$ & 0.383 \\
eGFR, mL/min/1.73 m ${ }^{2}$ & $98.50(63.65-126.40)$ & $110.15(86.90-124.43)$ & $0.043^{*}$ \\
Triglycerides, mmol/L & $1.59 \pm 0.90$ & $2.19 \pm 7.16$ & 0.586 \\
Cholesterol, mmol/L & $4.59(3.94-6.18)$ & $4.47(3.92-5.27)$ & 0.269 \\
LDL-C, mmol/L & $2.67(1.80-3.29)$ & $2.46(2.06-3.07)$ & 0.621 \\
Renal survival & & & \\
$\quad$ Complete remission (\%) & $12(27.3)$ & $37(25.2)$ & 0.779 \\
$\quad$ Partial remission (\%) & $9(20.5)$ & $55(37.4)$ & $0.037^{*}$ \\
$\quad$ Active renal disease (\%) & $13(29.5)$ & $39(26.5)$ & 0.693 \\
$\quad$ Renal insufficiency (\%) & $10(22.7)$ & $16(10.9)$ & $0.044^{*}$ \\
\hline
\end{tabular}

eGFR, estimated glomerular filtration rate; HPF, high-power field; LDL-C, low-density lipoprotein cholesterol; NS-HSPN, Henoch-Schönlein purpura nephritis presenting with nephrotic syndrome; SCr, serum creatinine; u-RBCs, erythrocytes in urine. ${ }^{*} p<0.05$.

Fig. 2. Kaplan-Meier analysis for the probability of composite endpoint, which was a $50 \%$ decline in estimated glomerular filtration rate, end-stage renal disease, and/or death. NS-HSPN, HenochSchönlein purpura nephritis presenting with nephrotic syndrome.

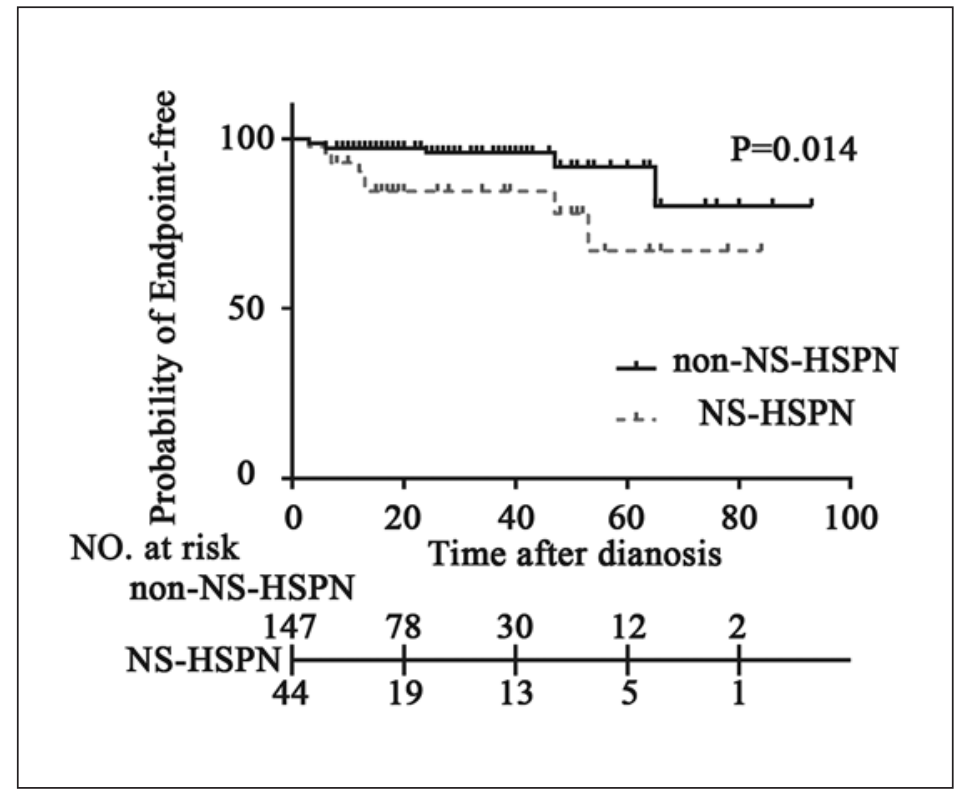

endpoints, as presented in Table 5. The results revealed that hypertension (HR 9.615, 95\% CI 1.398-66.131, $p<0.05$ ), eGFR (HR 1.040, 95\% CI 0.999-1.082, $p=0.058$ ), cystatin (HR 9.403, $95 \%$ CI 1.465-60.334, $p<0.05$ ) at onset, and tubular atrophy/interstitial fibrosis (HR 10.218, 95\% CI 1.398-66.131, $p<0.05$ ) in histopathological features were independent risk factors, and bloody stool (HR 5.402, 95\% CI 0.828-35.24, $p=0.078$ ) also seemed to be associated with poor outcome, although statistical significance was not found. Nevertheless, sex, age, gross hematuria, edema, treatment strategies, creatinine, and uric acid at onset were not correlated with renal outcome $(p>0.1)$. 
Table 5. Cox proportional hazard model of factors associated with renal insufficiency/ESRD in patients with HSPN

\begin{tabular}{lllllllll}
\hline & \multicolumn{2}{l}{ Unadjusted } & & \multicolumn{2}{l}{ Adjusted } \\
\cline { 2 - 3 } & HR & $95 \%$ CI & $p$ values & & HR & $95 \%$ CI & $p$ values \\
\hline Bloody stool & 3.226 & $0.766-13.589$ & 0.11 & & 5.402 & $0.828-35.240$ & 0.078 \\
Hypertension & 6.068 & $1.206-30.518$ & 0.029 & & 9.615 & $1.398-66.131$ & $0.021^{*}$ \\
eGFR & 0.995 & $0.979-1.011$ & 0.532 & & 1.040 & $0.999-1.082$ & $0.058^{*}$ \\
Cystatin C & 2.258 & $1.021-4.997$ & 0.044 & & 9.403 & $1.465-60.334$ & $0.018^{*}$ \\
T & 5.626 & $1.263-25.052$ & 0.023 & & 10.218 & $1.398-66.131$ & $0.021^{*}$ \\
\hline
\end{tabular}

eGFR, estimated glomerular filtration rate; ESRD, end-stage renal disease; HSPN, Henoch-Schönlein purpura nephritis; T, tubular atrophy/interstitial fibrosis. ${ }^{*} p<0.05$.

\section{Discussion}

HSPN is a common vasculitis mostly affecting children, with a lower incidence in adults. Meanwhile, less attention has been paid to NS-HSPN and its features are still not quite clear. In order to provide further information of adult-onset HSPN, we prospectively studied patients with HSPN manifested by nephrotic state. It could be easily noted that patients with NS-HSPN were characterized by severe symptoms and poor laboratory findings. Comparable with those reported elsewhere, our results suggest that edema was a common sign of NS-HSPN and hyperlipidemia was another peculiarity, mainly manifested by increased levels of cholesterol and LDL-C instead of triglycerides; it was widely recognized that hypoproteinemia and lower levels of lipoprotein lipase were to blame [3]. Apparently, patients with NS-HSPN were more likely to suffer from anemia in our study, probably caused by transferrin loss and severe hematuria. Above all, our study found that $40.9 \%$ of NS-HSPN patients complained of abdominal pain and that $20.5 \%$ of NS-HSPN patients had bloody stools, suggesting that gastrointestinal impairment was more common and clinical manifestations are more serious in NS-HSPN.

In our study, histopathological changes were various and renal lesions were more severe in NS-HSPN. It was reported previously that mesangial injury with different degrees of hypercellularity was the basic pathological pattern in children with HSPN [15]. Our study drew a similar conclusion that the majority of renal impairment of HSPN was mesangial proliferation. Furthermore, as is known, crescents usually present the active factor of disease, and there is an important link between crescent formation and rapidly progressive glomerulonephritis. Our study demonstrated that NS-HSPN patients tended to have higher proportions of crescents, revealing that NS-HSPN was an active form of vasculitis. Generally, patients with crescent glomerulonephritis will progress to renal insufficiency rapidly if treated inadequately. Consequently, aggressive treatment is widely used to treat patients with crescent glomerulonephritis with acceptable efficacy, which is the main reason why crescents fail to be a predictor of poor prognosis in many studies, including ours $[4,16]$. Meanwhile, it could serve as a reminder that we should treat NS-HSPN patients with severe clinicopathological manifestation timely and radically.

However, it was worth mentioning that our study prominently illustrated the poor prognosis of HSPN, depending on clinicopathological presentation rather than on different therapies. The correlation between NS and unfavorable prognosis was not hard to find in this study. The percentage of renal insufficiency was distinctly higher in NS-HSPN patients, and 


\section{Kidney \\ Blood Pressure \\ Research}

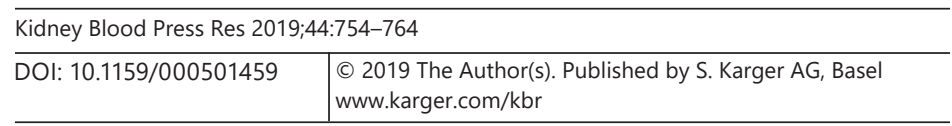

Tan et al.: The Nephrotic Syndrome of Henoch-Schönlein Purpura Nephritis

they were in a high-risk group that might progress to ESRD. The relationship has been extensively investigated in children by previous studies, and the conclusions that nephrotic state at onset can be regarded as a prognostic factor of adverse renal outcomes have been proposed [17-19]. Thus, more aggressive treatment regimens were tendentiously used to treat patients with NS-HSPN, which might partially explain why there were no differences in renal outcome by different therapies. Moreover, several studies have reported that aggressively immunosuppressive therapy has advantages as to decreasing proteinuria and that it is effective for treating NS-HSPN patients [20-22]. Nevertheless, immunosuppressive treatment was not a significant predictor in our study, and the data of our cohort illustrated that the poor prognosis of HSPN depended on clinicopathological presentation instead of treatment strategies. There is no denying that it is still controversial whether immunosuppressants are superior to glucocorticoids in the treatment of HSPN, which was not recommended by guidelines, and more high-quality research is required to solve this problem [23].

In addition, $7.9 \%$ of patients with HSPN in our cohort progressed to ESRD, and $18.2 \%$ of NS-HSPN patients reached the study endpoint. On the one hand, the prognosis of our HSPN patients seems to be better than that of Europeans, which might be caused by different races, geographical area, sample size, and other reasons [24-27]. On the other hand, although the fact that patients with NS-HSPN are more likely to progress to ESRD was proved in this study, there seems to be no significant differences in the rate of complete remission, implying that a discrepancy of efficacy existed in treating NS-HSPN. Hence, it is of great importance to find predictors of poor renal outcome, which is sure to benefit clinically and taken as a significant index in the treatment of NS-HSPN.

Thus, further investigation was carried out to explore the risk factors that contributed to NS-HSPN progression. Forty-four HSPN patients with nephrotic state at onset were assessed in isolation. The results of multivariate Cox regression analysis indicated that hypertension, eGFR, cystatin, and tubular atrophy/interstitial fibrosis can serve as independent risk factors in NS-HSPN. Notably, the prognostic indexes were not always consistent in the literature. Previous studies focused on pediatric patients with NS-HSPN proposed that the long-term outcomes principally based on clinical presentation and tubulointerstitial injuries were not relevant to renal outcome $[21,28]$. However, our research demonstrated that both clinical manifestation and pathological features could result in poor prognosis, which was consistent with studies whose objects were HSPN patients and that were not able to separate NS-HSPN from non-NS-HSPN $[5,16,25,27,29]$. This could be partially explained by two reasons. First, the timing of renal biopsies, different areas, and problems associated with recurrence or progression of NS-HSPN might play a part in the discrepancy. Second, it has been reported that adult and pediatric patients with HSPN have various clinical manifestations and histopathological findings and that adults tend to have worse renal outcome [25, 30]. Thus, we cannot afford to ignore patients of different ages. Surprisingly, we found that cystatin and renal outcome were interrelated in NS-HSPN. Cystatin C, abundantly expressed in extracellular fluids, is a 13-kDa subtype of cystatin with the function of inhibiting cysteine proteinase, which can be identified as a promising factor of renal insufficiency [31]. A recent cohort reported by Feng et al. [28] illustrated that children with NS-HSPN had higher cystatin levels. Another previous study revealed that cystatin was positively correlated with activity index and severity of pathological findings [32]. However, the role that cystatin plays in the pathogenic processes of HSPN is not elucidated yet, and in-depth studies are needed.

Some limitations of our study cannot be neglected. This was a single-center study with moderate sample size, and the patients enrolled were all Chinese. In addition, the follow-up of our study was not very long. All in all, a longer multicenter prospective study with a larger sample is required to help us achieve a better understanding of NS-HSPN in adults. 


\section{Conclusion}

The prognosis of adult patients with NS-HSPN, characterized by severe symptoms, poor laboratory findings, and histopathological changes, is worse than that of non-NS-HSPN patients. The unfavorable prognosis of HSPN principally depends on clinicopathological presentation rather than on different therapies, and hypertension, eGFR, cystatin, and tubular atrophy/interstitial fibrosis can serve as independent risk factors in NS-HSPN.

\section{Acknowledgments}

Sincere thanks go to Prof. Ping Fu, Baihai Su, Zhangxue Hu, Ye Tao, Lichuan Yang, Zi Li, and all the staff of the Department of Nephrology, West China Hospital.

\section{Statement of Ethics}

The subjects enrolled in our study gave their informed consent. This study was approved by the Ethics Committee of the West China Hospital of Sichuan University and fully complied with the Helsinki Declaration.

\section{Disclosure Statement}

The authors declare no conflicts of interest. This study received no funding.

\section{Author Contributions}

The study was designed by Wei Qin. The clinicopathological and follow-up data were collected by Jiaxing Tan, Yi Tang, Yicong Xu, Siyu Yan, Yuanyuan Xu, Li Tan, and Zhengxia Zhong. Data analysis was carried out by Jiaxing Tan, Li Tan, and Padamata Tarun. All authors listed significantly contributed to this paper. The authors declare that the paper is honest, accurate, and transparent.

\section{References}

1 Jennette JC, Falk RJ, Bacon PA, Basu N, Cid MC, Ferrario F, et al. 2012 revised International Chapel Hill Consensus Conference Nomenclature of Vasculitides. Arthritis Rheum. 2013 Jan;65(1):1-11.

2 Ozen S, Ruperto N, Dillon MJ, Bagga A, Barron K, Davin JC, et al. EULAR/PReS endorsed consensus criteria for the classification of childhood vasculitides. Ann Rheum Dis. 2006 Jul;65(7):936-41.

3 Hull RP, Goldsmith DJ. Nephrotic syndrome in adults. BMJ. 2008 May;336(7654):1185-9.

4 Shima Y, Nakanishi K, Sato M, Hama T, Mukaiyama H, Togawa H, et al. IgA nephropathy with presentation of nephrotic syndrome at onset in children. Pediatr Nephrol. 2017 Mar;32(3):457-65.

5 Pillebout E, Thervet E, Hill G, Alberti C, Vanhille P, Nochy D. Henoch-Schönlein Purpura in adults: outcome and prognostic factors. J Am Soc Nephrol. 2002 May;13(5):1271-8.

6 Mills JA, Michel BA, Bloch DA, Calabrese LH, Hunder GG, Arend WP, et al. The American College of Rheumatology 1990 criteria for the classification of Henoch-Schönlein purpura. Arthritis Rheum. 1990 Aug;33(8): 1114-21.

7 Tan J, Tang Y, Zhong Z, Yan S, Tan L, Tarun P, et al. The efficacy and safety of immunosuppressive agents plus steroids compared with steroids alone in the treatment of Henoch-Schönlein purpura nephritis: A metaanalysis. Int Urol Nephrol. 2019 Jun;51(6):975-85. 
Tan et al.: The Nephrotic Syndrome of Henoch-Schönlein Purpura Nephritis

8 Tan L, Tang Y, Peng W, Mathew BS, Qin W. Combined Immunosuppressive Treatment May Improve ShortTerm Renal Outcomes in Chinese Patients with Advanced IgA Nephropathy. Kidney Blood Press Res. 2018; 43(4):1333-43.

9 Kim CH, Lim BJ, Bae YS, Kwon YE, Kim YL, Nam KH, et al. Using the Oxford classification of IgA nephropathy to predict long-term outcomes of Henoch-Schönlein purpura nephritis in adults. Mod Pathol. 2014 Jul;27(7): 972-82.

10 Bellur SS, Roberts IS, Troyanov S, Royal V, Coppo R, Cook HT, et al. Reproducibility of the Oxford classification of immunoglobulin A nephropathy, impact of biopsy scoring on treatment allocation and clinical relevance of disagreements: evidence from the VALidation of IGA study cohort. Nephrol Dial Transplant. 2018 Dec. doi: 10.1093/ndt/gfy337 [Epub ahead of print].

11 Cattran DC, Coppo R, Cook HT, Feehally J, Roberts IS, Troyanov S, et al.; Working Group of the International IgA Nephropathy Network and the Renal Pathology Society. The Oxford classification of IgA nephropathy: rationale, clinicopathological correlations, and classification. Kidney Int. 2009 Sep;76(5):534-45.

12 Serriello I, Polci R, Feriozzi S, Gigante A, Di Giulio S, Rosa M, et al. Extracapillary proliferation is an independent predictive factor in Immunoglobulin A nephropathy. Nephrology (Carlton). 2015 Sep;20(9):654-9.

13 Zhang X, Shi S, Ouyang Y, Yang M, Shi M, Pan X, et al. A validation study of crescents in predicting ESRD in patients with IgA nephropathy. J Transl Med. 2018 May;16(1):115.

14 Mir S, Yavascan O, Mutlubas F, Yeniay B, Sonmez F. Clinical outcome in children with Henoch-Schönlein nephritis. Pediatr Nephrol. 2007 Jan;22(1):64-70.

15 Kawasaki Y, Ono A, Ohara S, Suzuki Y, Suyama K, Suzuki J, et al. Henoch-Schönlein purpura nephritis in childhood: pathogenesis, prognostic factors and treatment. Fukushima J Med Sci. 2013;59(1):15-26.

16 Huang X, Wu X, Le W, Hao Y, Wu J, Zeng C, et al. Renal Prognosis and Related Risk Factors for Henoch-Schönlein Purpura Nephritis: A Chinese Adult Patient Cohort. Sci Rep. 2018 Apr;8(1):5585.

17 Goldstein AR, White RH, Akuse R, Chantler C. Long-term follow-up of childhood Henoch-Schönlein nephritis. Lancet. 1992 Feb;339(8788):280-2.

18 Niaudet P, Habib R. Methylprednisolone pulse therapy in the treatment of severe forms of Schönlein-Henoch purpura nephritis. Pediatr Nephrol. 1998 Apr;12(3):238-43.

19 Schärer K, Krmar R, Querfeld U, Ruder H, Waldherr R, Schaefer F. Clinical outcome of Schönlein-Henoch purpura nephritis in children. Pediatr Nephrol. 1999 Nov;13(9):816-23.

20 Ren P, Han F, Chen L, Xu Y, Wang Y, Chen J. The combination of mycophenolate mofetil with corticosteroids induces remission of Henoch-Schönlein purpura nephritis. Am J Nephrol. 2012;36(3):271-7.

21 Wakaki H, Ishikura K, Hataya H, Hamasaki Y, Sakai T, Yata N, et al. Henoch-Schönlein purpura nephritis with nephrotic state in children: predictors of poor outcomes. Pediatr Nephrol. 2011 Jun;26(6):921-5.

22 Zhang Y, Gao Y, Zhang Z, Liu G, He H, Liu L. Leflunomide in addition to steroids improves proteinuria and renal function in adult Henoch-Schoenlein nephritis with nephrotic proteinuria. Nephrology (Carlton). 2014 Feb; 19(2): 94-100.

23 Chapter 11: Henoch-Schönlein purpura nephritis. Kidney Int Suppl (2011). 2012 Jun;2(2):218-20.

24 Calvo-Río V, Loricera J, Mata C, Martín L, Ortiz-Sanjuán F, Alvarez L, et al. Henoch-Schönlein purpura in northern Spain: clinical spectrum of the disease in 417 patients from a single center. Medicine (Baltimore). 2014 Mar;93(2):106-13.

25 Coppo R, Andrulli S, Amore A, Gianoglio B, Conti G, Peruzzi L, et al. Predictors of outcome in Henoch-Schönlein nephritis in children and adults. Am J Kidney Dis. 2006 Jun;47(6):993-1003.

26 Rauta V, Törnroth T, Grönhagen-Riska C. Henoch-Schoenlein nephritis in adults - clinical features and outcomes in Finnish patients. Clin Nephrol. 2002 Jul;58(1):1-8.

27 Shrestha S, Sumingan N, Tan J, Alhous H, McWilliam L, Ballardie F: Henoch Schönlein purpura with nephritis in adults: adverse prognostic indicators in a UK population. QJM. 2006 Apr;99(4):253-65.

28 Feng D, Huang WY, Hao S, Niu XL, Wang P, Wu Y, et al. A single-center analysis of Henoch-Schonlein purpura nephritis with nephrotic proteinuria in children. Pediatr Rheumatol Online J. 2017 Mar;15(1):15.

29 Ronkainen J, Nuutinen M, Koskimies O. The adult kidney 24 years after childhood Henoch-Schönlein purpura: a retrospective cohort study. Lancet. 2002 Aug;360(9334):666-70.

30 Lu S, Liu D, Xiao J, Yuan W, Wang X, Zhang X, et al. Comparison between adults and children with HenochSchönlein purpura nephritis. Pediatr Nephrol. 2015 May;30(5):791-6.

31 Roos JF, Doust J, Tett SE, Kirkpatrick CM. Diagnostic accuracy of cystatin C compared to serum creatinine for the estimation of renal dysfunction in adults and children - a meta-analysis. Clin Biochem. 2007 Mar;40(5-6): 383-91.

32 Lu S, Liu D, Xiao J, Cheng G, Zhang X, Liu Z, et al. Correlation Between Clinical and Pathological Characteristics of Henoch-Schönlein Purpura Nephritis in Adults. Iran J Kidney Dis. 2016 Jan;11(1):12-7. 